\title{
miR-345-3p serves a protective role during gestational diabetes mellitus by targeting BAK1
}

\author{
YUXIA LI $^{1}$ and JUN ZHUANG ${ }^{2}$ \\ ${ }^{1}$ Department of Gynecology and Obstetrics, Wuhan Children's Hospital, Wuhan Maternal and Child Healthcare \\ Hospital, Tongji Medical College, Huazhong University of Science and Technology, Wuhan, Hubei 430010; \\ ${ }^{2}$ Department of Obstetrics, Lianshui County People's Hospital, Huai'an, Jiangsu 223400, P.R. China
}

Received July 10, 2019; Accepted March 18, 2020

DOI: $10.3892 / \mathrm{etm} .2020 .9434$

\begin{abstract}
Recent studies have demonstrated that microRNAs (miRs) serve a crucial role during the development of gestational diabetes mellitus (GDM). However, the mechanisms underlying miR-345-3p and its protective role during GDM have not been previously reported. The present study investigated miR-345-3p expression and function in vitro, and the possible molecular mechanisms underlying GDM. Compared with healthy pregnant women, miR-345-3p was downregulated in the placental tissue and peripheral blood of patients with GDM. Further investigation revealed that BCL2-antagonist/killer 1 (BAK1) was a predicted target gene of miR-345-3p, and the expression of BAK1 was significantly increased in patients with GDM compared with healthy pregnant women. In vitro analysis revealed that miR-345-3p mimic significantly increased cell viability, migration and invasion, inhibited apoptosis, upregulated $\mathrm{Bcl}-2$ and matrix metallopeptidase 9 expression, and decreased Bax expression compared with the control group. Furthermore, miR-245-3p mimic-induced alterations were reversed by BAK1 overexpression. The results suggested that miR-345-3p overexpression exhibited a protective role in patients with GDM by inhibiting HTR8-/SVneo cell apoptosis, and promoting cell proliferation and migration via targeting BAK1. The use of miR-345-3p for the diagnosis of GDM requires further investigation.
\end{abstract}

\section{Introduction}

Gestational diabetes mellitus (GDM) is defined as any degree of glucose intolerance with onset or first recognition during pregnancy, excluding patients who exhibited diabetes prior to gestation but were first diagnosed during pregnancy (1).

Correspondence to: Mr. Jun Zhuang, Department of Obstetrics, Lianshui County People's Hospital, 6 Hongri Avenue, Lianchengzhen, Yucheng, Lianshui, Huai'an, Jiangsu 223400, P.R. China

E-mail: zhuangjun0107@163.com

Key words: microRNA-345-3p, gestational diabetes mellitus, BCL2-antagonist/killer 1, HTRB-/SVneo cells
GDM has become a major public health concern due to an increased prevalence, and the associated short- and long-term complications for the mother and the offspring (2). GDM is a multifactorial disease that is induced by environmental factors interacting with genes, and genetic factors are a major determinant of the disease (2). Additionally, the prevalence of GDM varies from country to country and region to region (3), with epidemiological studies indicating that the prevalence of GDM is $9 \%$ in the United States of America (3) compared with $3.0-21.2 \%$ in Asian countries (4). Therefore, the investigation of GDM has become worldwide in recent years (5); however, at present, the mechanisms underlying the development and progression of GDM are not completely understood.

GDM can alter the physiological condition of the mother; even when aggressively managed, GDM leaves its mark on offspring (6). In addition, the origin of the majority of GDM-associated adverse pregnancy outcomes involve the placenta (7). As a result, the placenta, which is the only interface that connects the mother and the fetus, has become an important organ for studying the pathogenesis of abnormal glucose metabolism, including GDM (7). Studies have indicated that the placenta exhibits immature placental villi or alterations in villus branch morphology during GDM, which lead to alterations in placental morphology and function, resulting in limited intrauterine growth and an increased risk of preterm birth (7). Therefore, chorionic trophoblast cells, which have invasive and endocrine functions, serve an important role during GDM (8). The active substances that are secreted by chorionic trophoblast cells, including hormones and neuropeptides, serve an important role during energy metabolism and transfer between mother and baby, maintenance of early pregnancy and trophic corpus luteum (9). The human chorionic trophoblast HTR8-/SVneo cell line is an immortalized cell line that was established and identified by a previous study (10). In the present study, the association between GDM and pathophysiology was assessed by determining the expression of miR-345-3p in the placenta and peripheral blood using in vitro experiments.

miRNAs are a class of highly evolutionarily conserved, single-chain, small-molecule, non-coding RNAs, measuring 18-23 nucleotides in length, that serve a regulatory role at the epigenetic level (11). Previous studies investigating miR-345 have focused on cancer, including prostate $(12,13)$ 
and colorectal cancer (14), as well as acute lymphocytic leukemia (15). Studies have identified miR-345 as a key regulator in a variety of types of cancer, primarily via its target genes and signaling pathways (12-16). miR-345 can also act as a tumor suppressor to affect cell epigenetic regulation, proliferation, apoptosis, differentiation, metabolism and chemosensitivity (17-19). Another study reported that miR-345 is differentially expressed during the differentiation of mouse C2C12 myoblasts into myotube differentiation port and umbilical cord mesenchymal stem cells (20). The aforementioned studies indicated that miR-345-3p serves an important regulatory role during cell growth, apoptosis, migration and invasion. Based on the key role of placental trophoblasts during the development and progression of GMD; it was hypothesized that miR-345-3p may be involved during the development and progression of GMD by regulating the biobehavior of placental trophoblast cells.

To explore the proposed hypothesis, the present study aimed to investigate the role and molecular mechanisms underlying miR-345-3p during GDM. The expression of miR-345-3p was measured in placental tissue samples from patients with GDM, and the regulatory association between miR-345-3p and BAK1 was assessed at the cellular level. The results of the present study suggested that miR-345-3p served a protective role during GDM by decreasing BAK1 expression; therefore, miR-345-3p may serve as a potential therapeutic target for human GDM. The results of the present study also highlighted the proapoptotic function of miR-345-3p during GDM, and supported its potential use as a diagnostic and therapeutic target for the disease.

\section{Materials and methods}

Clinical sample collection. A total of 30 blood samples and placental villous tissues were collected from 30 pregnant women with GDM (age range: 24-39 years) and 30 healthy pregnant women (age range: 24-39 years) form February 2017 to June 2018. Exclusion criteria were as follows: Age $<18$ years old; hypertensive disorder of pregnancy; other medical diseases during pregnancy (including pregnancy with diabetes); exposure to harmful substances during pregnancy; abnormal placenta or umbilical cord; maternal and child blood type incompatibility; and multiple pregnancies. Patients with GDM who did not fulfill any of the exclusion criteria were included in the present study. Both groups of pregnant women were hospitalized in Wuhan Children's Hospital. There were no significant differences in maternal birth, age, geographical origin or occupation between the two groups. The present study was approved by the Ethics Committee of Wuhan Children's Hospital (Wuhan Maternal and Child Healthcare Hospital). Written informed consent was obtained from all patients, and all patients approved the use of their blood samples and placental tissues in the present study.

GDM diagnostic criteria. Pregnant women at gestation week 24-28 were selected for the present study. Patients with GDM were diagnosed according to the American Diabetes Association standard for clinical diagnosis and treatment of diabetes (2011 edition) and standard oral glucose tolerance test (OGTT). The OGTT was performed as follows: Women fasted for 8-14 h or more, consumed sugar and subsequently consumed $75 \mathrm{~g}$ glucose powder dissolved in $200 \mathrm{ml}$ warm water within $5 \mathrm{~min}$. The blood glucose levels of the elbow vein were measured once every hour for $2 \mathrm{~h}$. The normal ranges for these aforementioned 3 blood glucose levels (including fasting level) are $>5.1,10.0$ and $8.5 \mathrm{mmol} / \mathrm{l}$, respectively. If any reading reached or exceeded the normal value, GDM was diagnosed.

Cell acquisition and culture. Human chorionic trophoblast cells (HTR8-/SVneo cells) were purchased from Shanghai Huzheng Industrial Co., Ltd. (cat no. HZ-CC337655). HTR8/SV-neo cells were routinely cultured in DMEM (Invitrogen; Thermo Fisher Scientific, Inc.) high sugar complete medium containing 10\% FBS (Invitrogen; Thermo Fisher Scientific, Inc.) with 5\% $\mathrm{CO}_{2}$ at $37^{\circ} \mathrm{C}$.

Reverse transcription-quantitative PCR (RT-qPCR). Total RNA was extracted from blood, tissues and cells using the TRIzol reagent (Thermo Fisher Scientific, Inc.) according to manufacturer's protocols. A cDNA Synthesis kit (Invitrogen; Thermo Fisher Scientific, Inc.) was used to perform RT according to the manufacturer's protocols. Reverse transcription reaction condition was as following: $25^{\circ} \mathrm{C}$ for $5 \mathrm{~min}, 42^{\circ} \mathrm{C}$ for $60 \mathrm{~min}$ and $80^{\circ} \mathrm{C}$ for $2 \mathrm{~min}$. qPCR analysis was performed using the LightCycler 480 SYBR Green I Master kit (Roche Applied Science) according to manufacturer's protocols and a LightCycler 480 II instrument (Roche Applied Science). The following thermocycling conditions were used for qPCR: Initial denaturation at $95^{\circ} \mathrm{C}$ for $5 \mathrm{~min}$; followed by 45 cycles of $94^{\circ} \mathrm{C}$ for $10 \mathrm{sec}$, primer pair-specific annealing at $55^{\circ} \mathrm{C}$ for $20 \mathrm{sec}$ and $72^{\circ} \mathrm{C}$ for $30 \mathrm{sec}$, followed by a final extension step at $72^{\circ} \mathrm{C}$ for $10 \mathrm{~min}$. mRNA and miRNA expression levels were quantified using the $2^{-\Delta \Delta \mathrm{Cq}}$ method (21), and normalized to the internal reference genes $\beta$-actin and U6, respectively. Primer sequences were as follows: miR-345-3p forward, 5'-GGTTTT TGGATTGGGTTGTAGAGTG-3' and reverse, 5'-AACCAA AACAATCCCTTACCACTAC-3'; BAK1 forward, 5'-GCT CCCAACCCATTCACTAC-3' and reverse, 5'-TCCCTACTC CTTTTCCCTGA-3'; U6 forward, 5'-GCTTCGGCAGCA CATATACTAAAAT-3' and reverse, 5'-CGCTTCACGAAT TTGCGTGTCAT-3'; GAPDH forward, 5'-CTTTGGTATCGT GGAAGGACTC-3' and reverse, 5'-GTAGAGGCAGGGATG ATGTTCT-3'.

Identification of miR-345-3p target BAK1. TargetScan (version 7.2; www.targetscan.org/vert_72) was used to predict the target gene of miR-345-3p and to identify target gene 3'-untranslated region (UTR) specific binding sites, in combination with the National Center for Biotechnology Information database (https://www.ncbi.nlm.nih.gov/). The binding sites between miR-345-3p and 3'UTR BAK1 were observed. Subsequently, a dual-luciferase reporter assay was performed to investigate whether miR-345-3p directly interacted with BAK1.

Dual-luciferase reporter assay. Wild-type (WT-BAK1) and mutant (MUT-BAK1) 3'UTR BAK1 were cloned into pmiR-RB-Report ${ }^{\mathrm{TM}}$ dual luciferase reporter gene plasmid vectors (Guangzhou RiboBio Co., Ltd.), according to the 
manufacturer's protocol. 293T cells $\left(5 \times 10^{4}\right.$ cells per well; American Type Culture Collection) were co-transfected with WT-BAK1 or MUT-BAK1, and miR-345-3p mimic or mimic control using Lipofectamine ${ }^{\circledR} 2000$ (Invitrogen; Thermo Fisher Scientific, Inc.) according to the manufacturer's protocol. Following incubation at $37^{\circ} \mathrm{C}$ for $48 \mathrm{~h}$, luciferase activities were detected using a Dual-luciferase assay system (Promega Corporation), according to the manufacturer's protocol. Firefly luciferase activity was normalized to Renilla luciferase activity.

Cell transfection. Cells were seeded into a six well plate ( $5 \times 10^{4}$ cells per well) and allowed to reach $80-90 \%$ confluence. HTRB-/SVneo cells were transfected with $1 \mu \mathrm{g}$ BAK1-plasmid (Cat no. sc-400646-ACT; Santa Cruz Biotechnology, Inc.), $1 \mu \mathrm{g}$ control-plasmid (cat no. sc-437275; Santa Cruz Biotechnology, Inc.), $50 \mathrm{nM}$ miR-345-3p mimic (HmiR0210-MR03; GeneCopoeia), $50 \mathrm{nM}$ mimic control (cat. no. CmiR0001-MR03; GeneCopoeia), $1 \mu \mathrm{g}$ control-plasmid $+50 \mathrm{nM} \mathrm{miR}-345-3 \mathrm{p}$ mimic or $1 \mu \mathrm{g}$ BAK1-plasmid $+50 \mathrm{nM}$ miR-345-3p mimic using Lipofectamine $^{\circledR} 2000$ (Invitrogen; Thermo Fisher Scientific, Inc.), according to the manufacturer's protocol. Following incubation for $48 \mathrm{~h}$, transfection efficiency was determined by RT-qPCR.

Cell viability. Cells in the logarithmic growth phase were plated ( $5 \times 10^{4}$ cells per well) into a 96-well plate. At $\sim 90 \%$ confluence, HTR8-/SVneo cells were transfected with miR-345-3p mimic, mimic control, control-plasmid + miR-345-3p mimic or BAK1-plasmid + miR-345-3p mimic for $48 \mathrm{~h}$ at $37^{\circ} \mathrm{C}$. Subsequently, $20 \mu \mathrm{l}$ MTT solution $(5 \mathrm{mg} / \mathrm{ml})$ was added to each well and cultured at $37^{\circ} \mathrm{C}$ for $4 \mathrm{~h}$. The solution was discarded and DMSO $(150 \mu \mathrm{l})$ was added to each well at $37^{\circ} \mathrm{C}$ for $10 \mathrm{~min}$ with gentle agitation to sufficiently dissolve the purple formazan. The optical density (OD) value of each well was measured at a wavelength of $562 \mathrm{~nm}$ using a microplate reader. Cell viability was calculated as follows: (Experimental group OD value/control OD value) x100\%. A total of 6 wells were used for each group and the experiment was performed in triplicate.

Detection of apoptosis by flow cytometry. At $48 \mathrm{~h}$ post-transfection, cell apoptosis was determined using the AnnexinV-FITC/PI kit [cat no 70-AP101-100; Hangzhou Multi Sciences (Lianke) Biotech Co., Ltd.], according to the manufacturer's protocol. Early and late apoptosis were detected using a FACSCalibur flow cytometer (BD Biosciences) with FlowJo software (version 7.2.4; FlowJo LLC).

Transwell assay for the detection of cell migration and invasion. Non-Matrigel-coated and Matrigel-coated Boyden chambers (BD Biosciences) were used to detect the migratory and invasive abilities of HTRB-/SVneo cells, respectively. HTRB-/SVneo cells were transfected with miR-345-3p mimic, mimic control, control-plasmid + miR -345-3p mimic or BAK1-plasmid + miR-345-3p mimic for $48 \mathrm{~h}$. Following starvation overnight in serum-free DMEM at $37^{\circ} \mathrm{C}$ for $24 \mathrm{~h}$, cells were obtained by $0.25 \%$ trypsinization. The cells $\left(5 \times 10^{6}\right.$ cells $\left./ \mathrm{ml}\right)$ in serum-free medium were plated in the upper chambers of the Transwell plates. DMEM containing $2.5 \%$ FBS was plated into the lower chambers of the Transwell plates. After incubation at $37^{\circ} \mathrm{C}$ for $24 \mathrm{~h}$, cells remaining on the upper chamber surface were wiped off using a cotton swab. Subsequently, the upper chamber of the Transwell insert was washed with PBS, and cells on the lower surface of the Transwell chamber were fixed with $4 \%$ paraformaldehyde at room temperature for $30 \mathrm{~min}$. Then the cells were stained using $0.1 \%$ crystal violet (Beyotime Institute of Biotechnology) for $20 \mathrm{~min}$ at room temperature. The number of migratory cells was counted under a light microscope (magnification, $\mathrm{x} 40$ ) in 5 fields of view, and the total number of cells was recorded and the mean number of migratory cells was calculated.

Western blotting. Total protein from HTRB-/SVneo cells was extracted using RIPA buffer (Beyotime Institute of Biotechnology) at $4^{\circ} \mathrm{C}$ for $1 \mathrm{~h}$. The protein concentration was determined with a bicinchoninic acid assay kit (Beyotime Institute of Biotechnology). Proteins (30 $\mu \mathrm{g} /$ lane) were separated via $10 \%$ SDS-PAGE and transferred onto PVDF membranes. Subsequently, the membranes were blocked with 5\% skim milk in TBS containing Tween 20 (TBST) at room temperature for $1.5 \mathrm{~h}$. The membranes were incubated with primary antibodies targeted against: Bcl-2 (Cat no. sc-23960; 1:1,000; Santa Cruz Biotechnology, Inc.), Bax (Cat no. sc-20067; 1:1,000; Santa Cruz Biotechnology, Inc.), matrix metallopeptidase (MMP)9 (Cat no. ab38898; 1:1,000; Abcam) and GAPDH (Cat no. Ab181602; 1:1,000; Abcam). After washing with TBST, the membrane was incubated with the corresponding horseradish peroxidase-labeled secondary antibody (1:2,000; cat no. 7074; Cell Signaling Technology, Inc.) at room temperature for $2 \mathrm{~h}$ at room temperature. Protein bands were visualized using enhanced chemiluminescence kit (Applygen Technologies, Inc.). Protein expression levels were quantified using ImageJ software (version 1.46; National Institutes of Health) with GAPDH as the loading control.

Statistical analysis. Statistical analyses were performed using SPSS software (version 18.0; SPSS, Inc.). Data are presented as the mean \pm standard deviation. One-way ANOVA followed by a Tukey's post hoc test was used to analyze the differences among multiple groups. The unpaired Student's t-test was used to analyze the statistical significance between two groups. $\mathrm{P}<0.05$ was considered to indicate a statistically significant difference.

\section{Results}

Expression of miR-345-3p in the peripheral blood and placental tissues of patients with GDM. RT-qPCR was used to detect miR-345-3p expression levels in maternal placental tissues and peripheral blood of patients with GDM and healthy control subjects. miR-345-3p expression was significantly decreased in the placental tissues and peripheral blood of patients with GDM compared with normal pregnant women (Fig. 1).

$B A K 1$ is the target gene of $m i R-345-3 p$. The target gene of miR-345-3p was predicted using TargetScan. The results indicated that miR-345-3p had hundreds of potential target genes, 


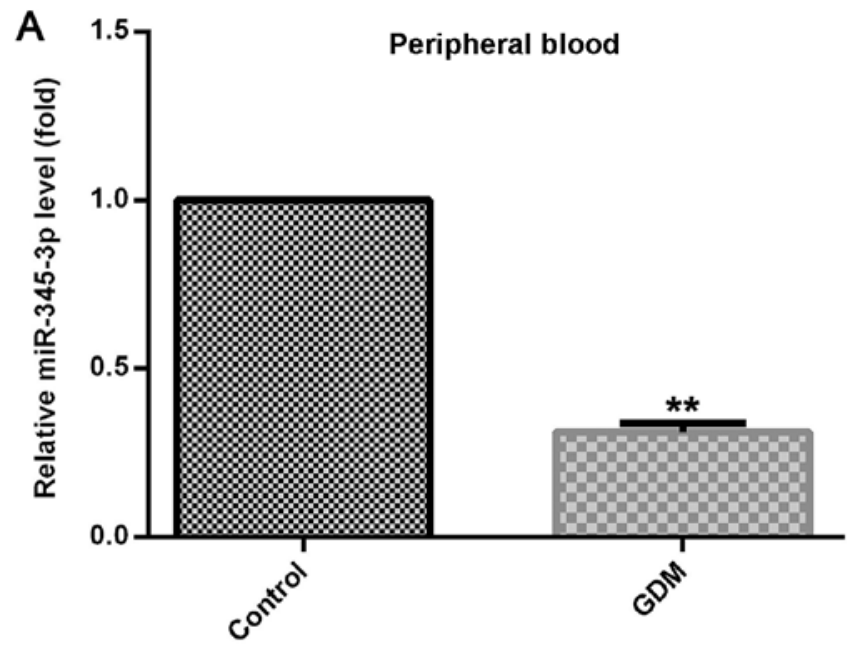

B

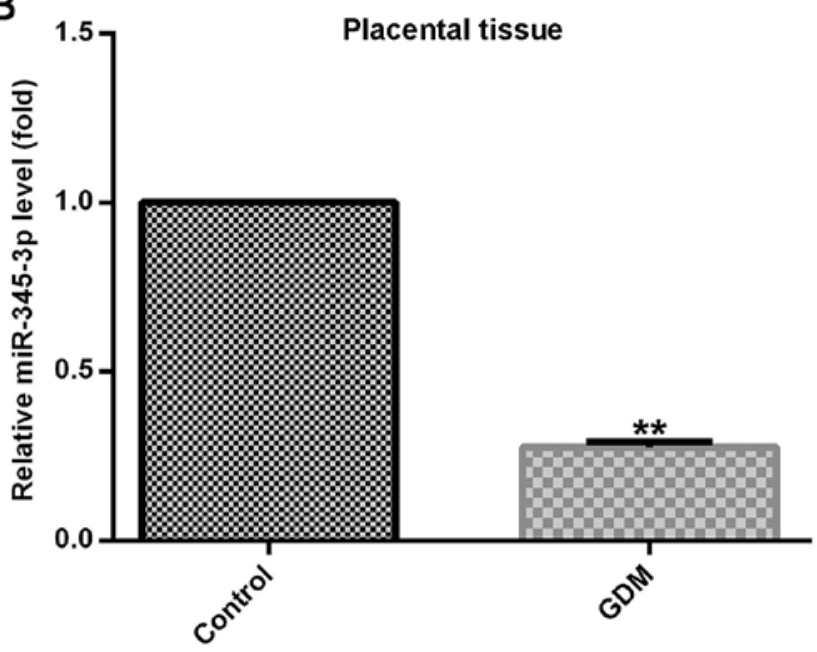

Figure 1. Differential expression of miR-345-3p in the peripheral blood and placental tissues of normal pregnant women and patients with GDM. miR-345-3p mRNA expression levels in the (A) peripheral blood and (B) placental tissues of normal pregnant women and patients with GDM. ${ }^{* *} \mathrm{P}<0.01$ vs. control. miR, microRNA; GDM, gestational diabetes mellitus.

including BAK1 (Fig. 2A). The BAK1 protein belongs to the BCL2 protein family, and localizes to mitochondria to induce apoptosis (22). As placental trophoblast growth serves a key role during the development and progression of GDM (23), it was hypothesized that BAK1 may serve important functions during the growth of placental trophoblast cells. Moreover, the role of BAK1 during GDM is not completely understood; therefore, BAK1 was selected for further investigation in the present study.

A dual-luciferase reporter assay was performed to verify that BAK1 was a target gene of miR-345-3p. miR-345-3p mimic significantly decreased the luciferase activity of $293 \mathrm{~T}$ cells transfected with BAK1-WT compared with the mimic control group, while miR-345-3p mimic displayed no significant effect on the luciferase activity of HTRB-/SVneo cells transfected with BAK1-MUT. The results indicated that BAK1 was a target of miR-345-3p (Fig. 2B).

Expression of BAK1 in the peripheral blood and placental tissues of patients with GDM. RT-qPCR was performed to determined BAK1 mRNA expression levels in the maternal placental tissues and peripheral blood of patients with GDM and the normal pregnancy control group. The BAK1 mRNA expression levels were significantly increased in the maternal peripheral blood and placental tissues of the GDM group compared with the control group (Fig. 2C and D).

Negative regulation of BAK1 by $\mathrm{miR}-345-3 p$ in $H T R B-/ S V n e o$ cells. To investigate the effect of miR-345-3p on BAK1 expression, HTRB-/SVneo cells were transfected with BAK1-plasmid, control-plasmid, miR-345-3p mimic, mimic control, control-plasmid + miR-345-3p mimic or BAK1-plasmid + miR-345-3p mimic for $48 \mathrm{~h}$. The expression of miR-345-3p in the miR-345-3p mimic group was significantly increased compared with the control group (Fig. 3A). BAK1 mRNA expression was increased in the BAK1-plasmid group compared with the control group (Fig. 3B). In addition, BAK1 expression was significantly decreased in the miR-345-3p mimic group compared with the control group; however, co-transfection with BAK1-plasmid reversed the miR-345-3p mimic-induced decreased in BAK1 expression (Fig. 3C and D).

Effect of miR-345-3p on HTRB-/SVneo cells. In the present study, the effects of miR-345-3p on HTRB-/SVneo cells were assessed by detecting cell viability, apoptosis, migration and invasion, as well as detecting the expression levels of Bcl-2, Bax and MMP-9. Compared with the control group, miR-345-3p mimic significantly enhanced HTRB-/SVneo cell viability (Fig. 4A), migration (Fig. 4B) and invasion (Fig. 4C), and significantly inhibited cell apoptosis (Fig. 4D and E). Transfection with the BAK1-plasmid significantly reversed the miR-345-3p mimic-mediated effects on HTRB-/SVneo cell activity.

Furthermore, miR-345-3p mimic significantly enhanced the protein expression levels of Bcl-2 (Fig. 5A and $\mathrm{B}$ ) and MMP-9 (Fig. 5A and C), and significantly decreased the protein expression levels of Bax (Fig. 5A and D) in HTRB-/SVneo cells compared with the control group. Similarly, miR-345-3p mimic-induced alterations to protein expression levels were significantly reversed by BAK1-plasmid.

\section{Discussion}

GDM is one of the most common complications that occurs during pregnancy. The disease has a multi-sourced etiology (3), which may be the result of a combination of genetic and environmental factors; however, the pathogenesis of GDM is not completely understood. A number of studies have demonstrated that miRNAs are ubiquitously expressed in various organisms and exhibit biological characteristics, including highly conserved and time-dependent expression, as well as tissue expression specificity $(11,24,25)$. miRNAs serve a post-transcriptional regulatory role by binding to the 3'UTR of mRNAs. Li et al (26) conducted an miRNA microarray analysis that indicated that 9 miRNAs were significantly altered in GDM samples: miR-508-3p was upregulated, and miR-27a, miR-9, miR-30d, miR-362-5p, miR-137, miR-92a, miR-33a and miR-502-5p were downregulated, suggesting that miRNA may serve a regulatory role during the development of GDM. Therefore, investigating the expression and 

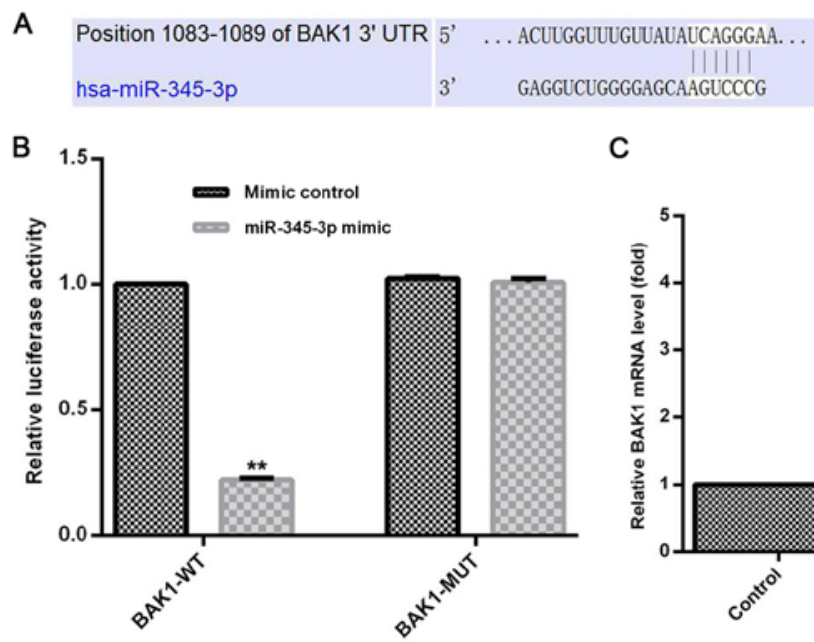

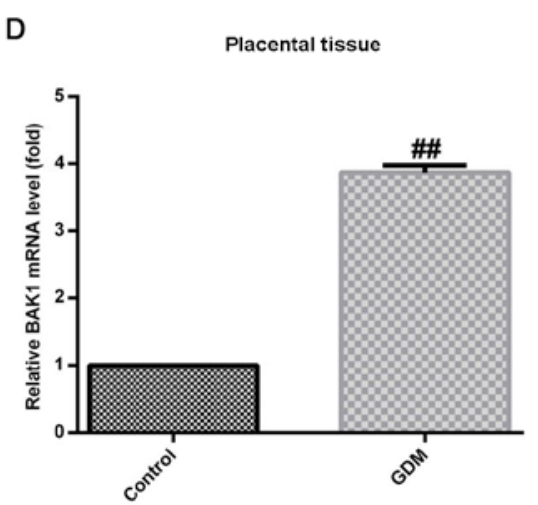

Figure 2. Prediction and verification of the target gene of miR-345-3p in HTRB-/SVneo cells, and the differential expression of BAK1 in the peripheral blood and placental tissues of normal pregnant women and patients with GDM. (A) TargetScan was used to predict the binding sites between BAK1 and miR-345-3p. (B) A dual-luciferase reporter assay was performed to verify the binding sites between BAK1 and miR-345-3p. (C and D) BAK1 mRNA expression levels in the $(\mathrm{C})$ peripheral blood and (D) placental tissues of normal pregnant women and patients with GDM. ${ }^{* *} \mathrm{P}<0.01$ vs. mimic control. ${ }^{\# \#} \mathrm{P}<0.01$ vs. control. miR, microRNA; BAK1, BCL2-antagonist/killer 1; GDM, gestational diabetes mellitus; 3'UTR, 3'-untranslated region; WT, wild-type; MUT, mutant.

A

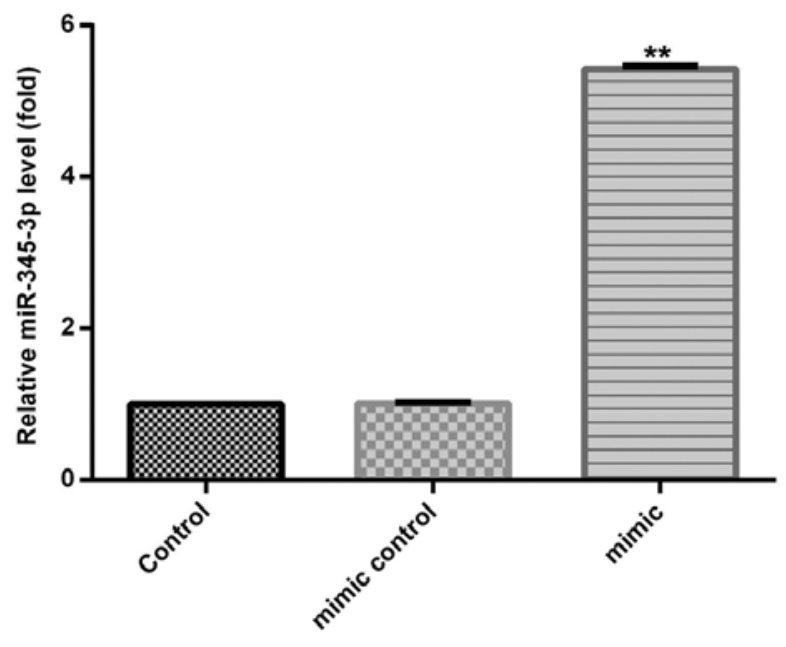

C

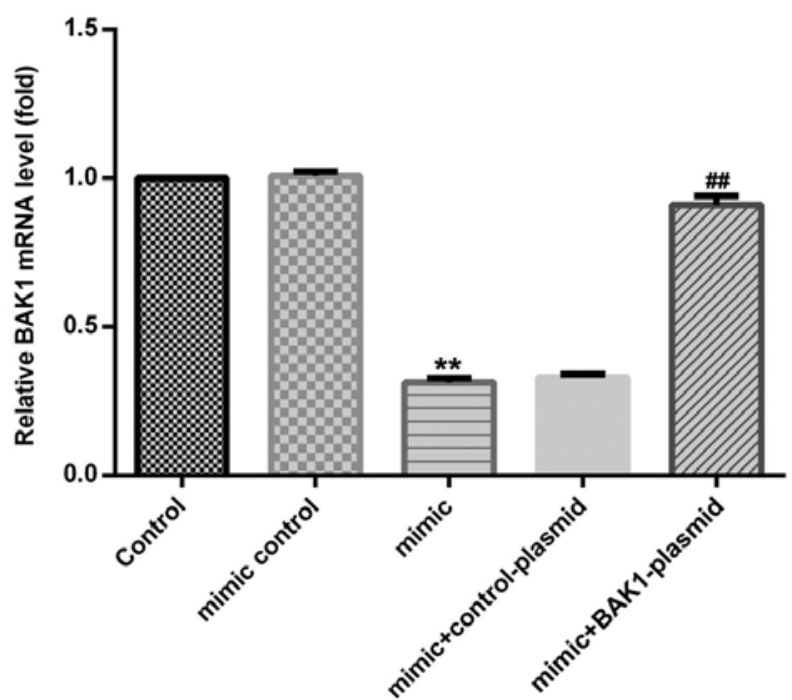

B

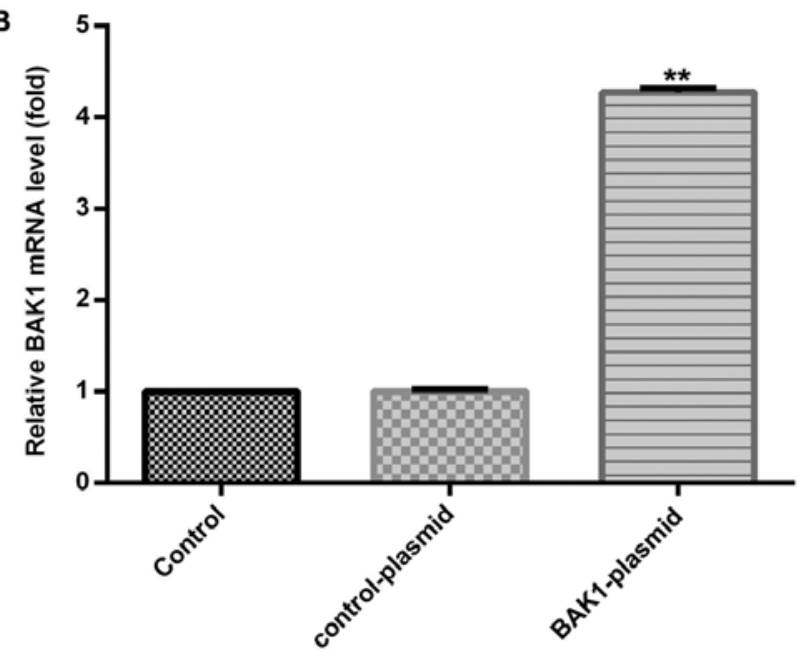

D

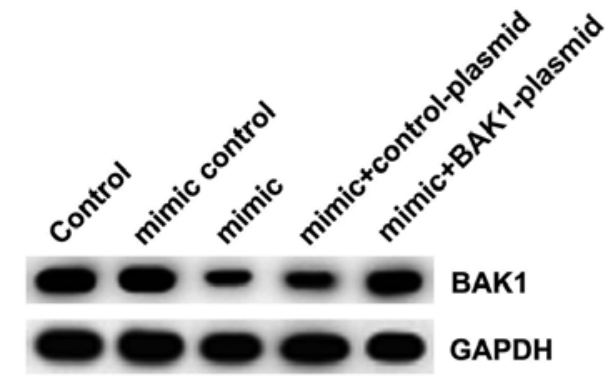

Figure 3. Effect of miR-345-3p on BAK1 expression in HTRB-/SVneo cells. (A) miR-345-3p expression levels in HTRB-/SVneo cells transfected with miR-345-3p mimic or mimic control. (B) BAK1 mRNA expression levels in HTRB-/SVneo cells transfected with BAK1-plasmid or control-plasmid. (C) mRNA and (D) protein expression levels of BAK1 in HTRB-/SVneo cells transfected with mimic control, miR-345-3p mimic, miR-345-3p mimic + control-plasmid or miR-345-3p mimic + BAK1-plasmid. ${ }^{* *} \mathrm{P}<0.01$ vs. control. ${ }^{\# /} \mathrm{P}<0.01$ vs. mimic. miR, microRNA; BAK1, BCL2-antagonist/killer 1. 

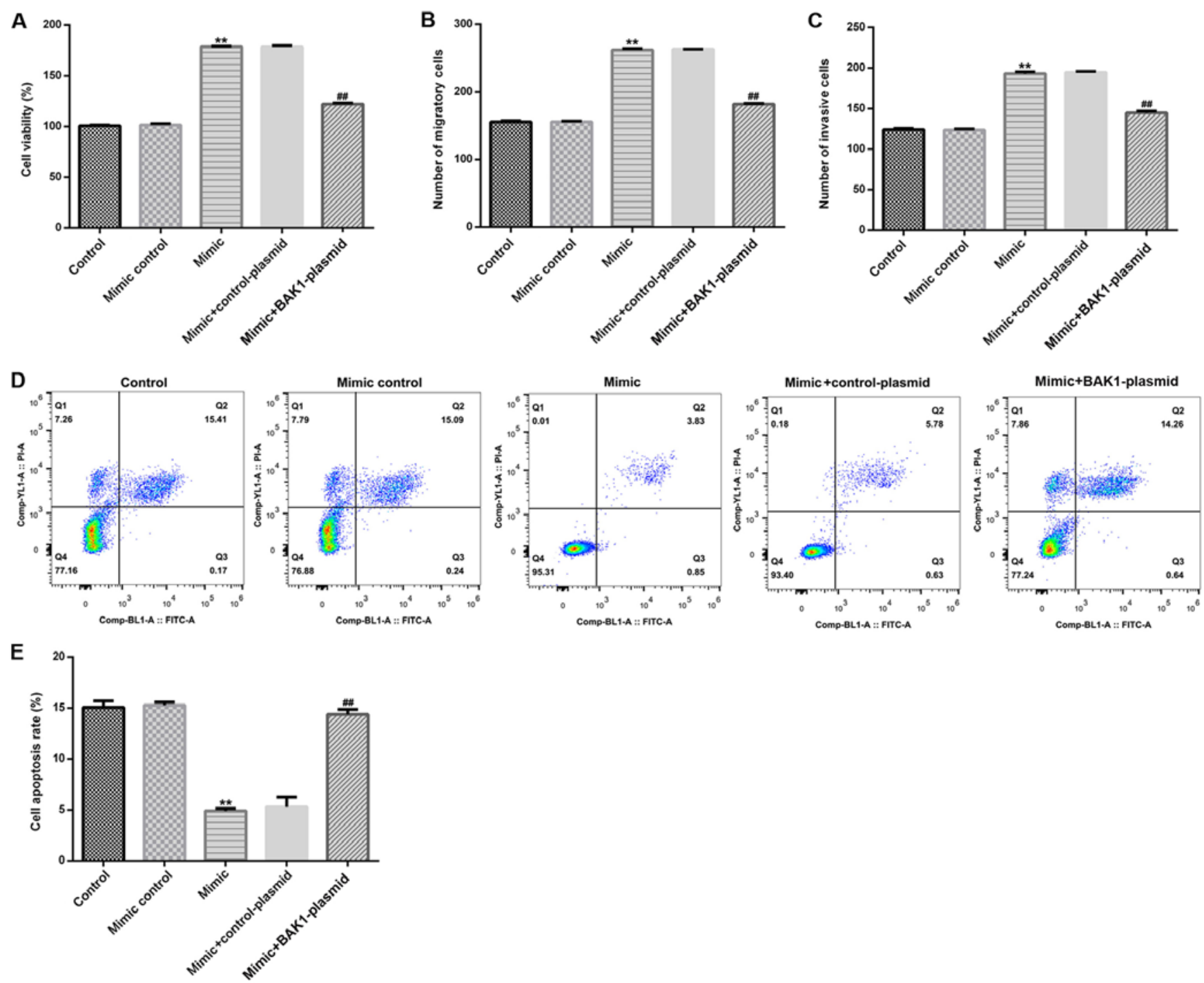

Figure 4. Effect of miR-345-3p on HTRB-/SVneo cells. HTRB-/SVneo cells were transfected with miR-345-3p mimic, mimic control, miR-345-3p mimic + control-plasmid or BAK1-plasmid + miR-345-3p mimic for $48 \mathrm{~h}$. (A) The MTT assay was performed to determine cell viability. Transwell assays were performed to determine cell (B) migration and (C) invasion. (D) Early and late cell apoptosis was determined by flow cytometry. (E) Quantification of the flow cytometry data. ${ }^{* *} \mathrm{P}<0.01$ vs. control. ${ }^{\# \#} \mathrm{P}<0.01$ vs. mimic. miR, microRNA; BAK1, BCL2-antagonist/killer 1 .

regulatory mechanisms underlying miRNAs in patients with GDM is required to provide novel insight into the pathogenesis of GDM.

To investigate the effect and mechanisms underlying miR-345-3p during GDM, the expression of miR-345-3p was measured in the peripheral blood and placental tissues of patients with GDM and healthy pregnant women using RT-qPCR. The results indicated that the expression of miR-345-3p in the peripheral blood and placental tissues of patients with GDM was significantly decreased compared with healthy pregnant women, indicating that low-level miR-345-3p expression may be associated with GDM pathophysiology. In addition, miRNAs have been reported to serve an important role during diabetes $(11,27,28)$. Monfared et al (11) demonstrated the extensive role of miR-135a as a gene regulator following GDM transplantation, which suggested that miR-135a may serve as a potential indicator for the prevention, treatment and management of GDM. The serum miRNA pattern of type 1 diabetes and the signaling pathways that may be associated with its pathogenesis have been previously identified (27). Erener et al (27) reported that miR-29b is highly expressed in retinal ganglion cell neurons in a rat model of diabetes, and indicated that miR-29b may be associated with the pathogenesis of diabetic retinopathy. Additionally, miR-29b has also been associated with the progression of renal fibrosis, including diabetic nephropathy (29). The aforementioned studies indicated that miRNAs may be associated with the pathogenesis of diabetes.

miRNAs are involved in a number of cellular biological processes by regulating post-transcriptional expression levels, either by degrading target genes or inhibiting the translation of target genes. To identify the biological functions of miRNAs, screening and validation of miRNA target genes has become a research focus. Therefore, the present study used HTRB-/SVneo cells to investigate the target genes of miR-345-3p, predicting potential target genes via TargetScan and verifying these target genes using a dual-luciferase reporter assay. The results indicated that BAK1 was a target 
A

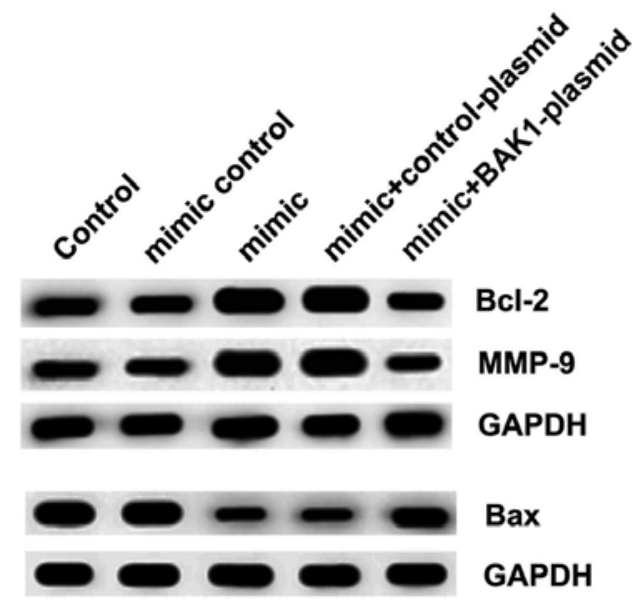

C

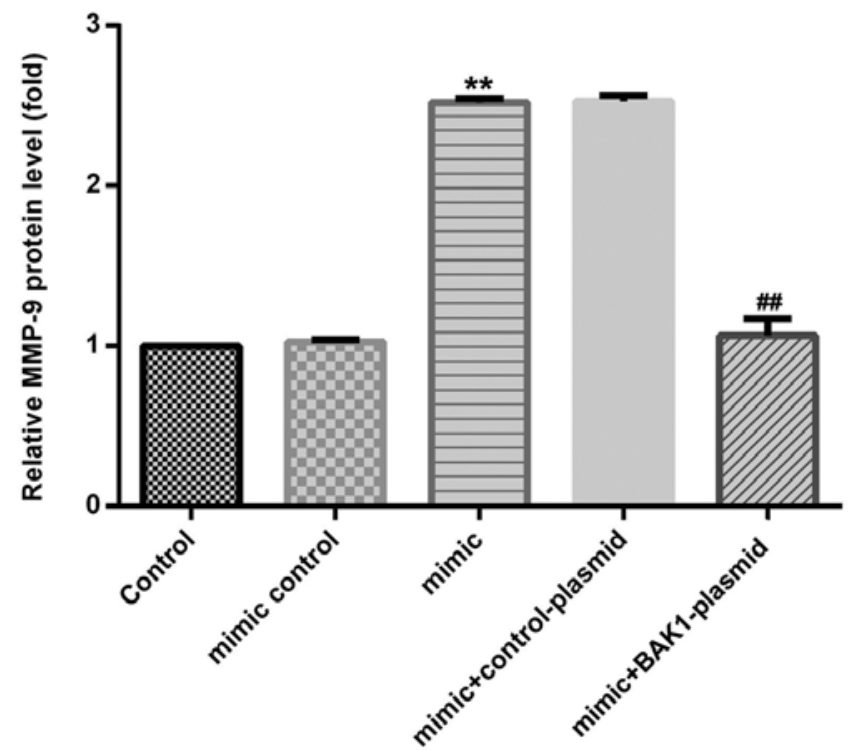

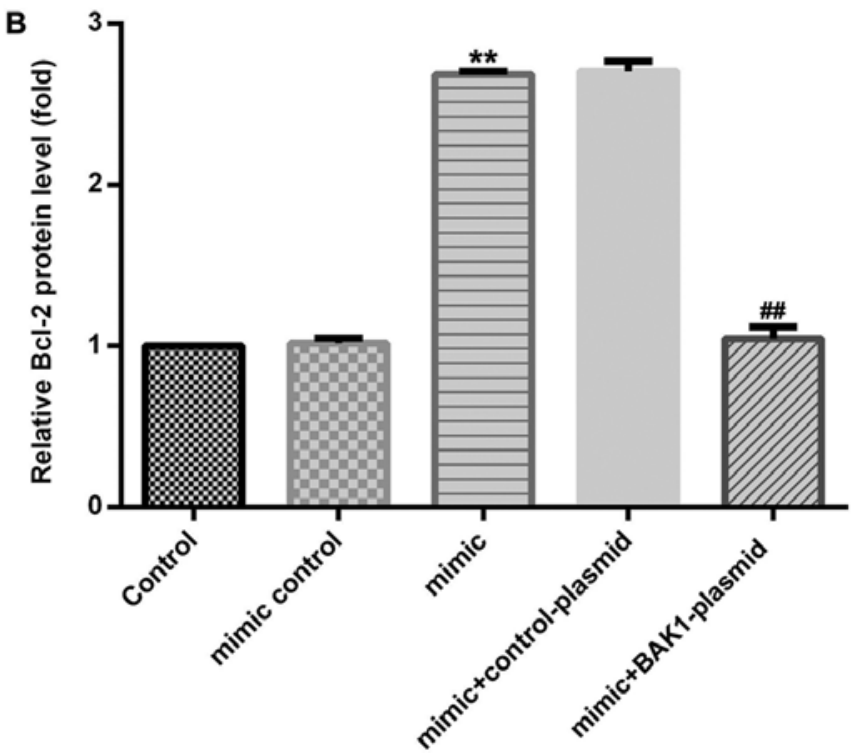

D

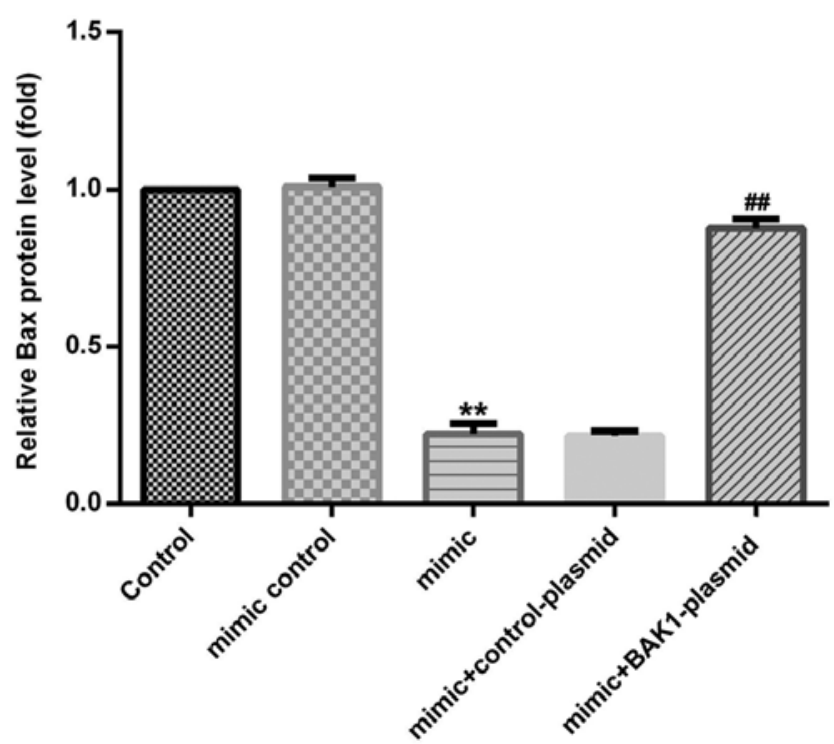

Figure 5. Effect of miR-345-3p on the expression of Bcl-2, MMP-9 and Bax in HTRB-/SVneo cells. HTRB-/SVneo cells were transfected with miR-345-3p mimic, mimic control, miR-345-3p mimic + control-plasmid or BAK1-plasmid + miR-345-3p mimic for $48 \mathrm{~h}$. (A) Protein expression levels were determined by western blotting. (B-D) Quantified densitometric data for (B) Bcl-2, (C) MMP-9 and (D) Bax protein expression levels. ${ }^{* *} \mathrm{P}<0.01$ vs. control. ${ }^{* \#} \mathrm{P}<0.01$ vs. mimic. miR, microRNA; MMP-9, matrix metallopeptidase 9; BAK1, BCL2-antagonist/killer 1.

gene of miR-345-3p. Subsequently, whether BAK1 was expressed in the peripheral blood and placental tissues of patients with GDM was assessed. The expression of BAK1 in the peripheral blood and placental tissues of patients with GDM was detected using RT-qPCR. The results indicated that BAK1 expression was significantly increased in the peripheral blood and placental tissues of patients with GDM compared with healthy pregnant women.

BAK1 was expressed in patients with GDM; therefore, the association between BAK1 and miR-345-3p was assessed in HTRB-/SVneo cells. Transfection of HTRB-/SVneo cells with BAK1-plasmid, control-plasmid, miR-345-3p mimic, mimic control or BAK1-plasmid + miR-345-3p mimic for $48 \mathrm{~h}$ indicated that miR-345-3p mimic significantly decreased BAK1 expression compared with the control group. miR-345-3p mimic-mediated effects on BAK1 expression were reversed by co-transfection with BAK1-plasmid. Therefore, the results suggested that miR-345-3p exhibited a negative regulatory effect on BAK1. MTT, flow cytometry, Transwell assay and western blot analyses were subsequently performed to investigate the effects of BAK1 expression on miR-345-3p-mediated cellular functions in HTRB-/SVneo cells, including cell viability, apoptosis, migration and invasion. miR-345-3p mimic significantly increased HTRB-/SVneo cell viability, migration and invasion, and inhibited cell apoptosis compared with the control group. The miR-345-3p-mediated effects on cellular functions were reversed by BAK1 overexpression. In addition, transfection with the miR-345-3p mimic significantly increased the expression of Bcl-2 and MMP9 in HTRB-/SVneo cells, and decreased the expression of 
Bax, which was also reversed by BAK1 overexpression. Cell functions, including cell viability, migration, invasion and apoptosis are closely associated with cell differentiation, tissue and organ development (30), indicating that miR-345-3p may serve an important regulatory role during the development and function of the placenta during embryonic development. In the present study, miR-345-3p expression levels were significantly decreased in the placental tissues of patients with GDM compared with normal pregnant women, which suggested that GDM may be associated with dysfunction of trophoblast cell migration and invasion. Therefore, during GDM, miR-345-3p downregulation in placental trophoblast cells may increase the expression of downstream target genes, thereby affecting trophoblast cell proliferation, viability and apoptosis. Alterations to cellular function lead to abnormalities in placental structure and function, particularly placental invasion and secretion, during embryonic development $(31,32)$.

The results of the present study indicated that HTRB-/SVneo cell functions were sensitive to high levels of miR-345-3p. Among the HTRB-/SVneo cell functions evaluated in the present study, migration, invasion and cell viability were enhanced by miR-345-3p over-expression, which is important for the molecular pathogenesis of GDM (23). Invasion is an important function of trophoblast cells $(33,34)$, with adhesion and invasion of the maternal uterine epithelium being the premise of placenta formation (35). Insufficient extravillous trophoblast invasion of the endometrium has been reported to cause pregnancy-associated complications, including miscarriage, pregnancy-induced hypertension and fetal growth restriction (36). Moreover, the migration and invasion of trophoblast cells has been suggested to be associated with insulin resistance (37). The results of the present study suggested that miR-345-3p expression decreased in GDM maternal placenta tissue and blood. miR-345-3p upregulation increased the migration and invasion abilities of placental trophoblast cells, which may be one of the causes of GDM. Previous study has indicated that the high glucose environment in pregnant women with GDM affects trophoblast cell proliferation, apoptosis and cell cycle regulation (38). The expression of the target gene BAK1 was increased in patients with GDM compared with normal pregnant women, suggesting that BAK1 promoted the migration and invasion of trophoblast cells, and potentially induced the high glucose environment. The present study provided novel insight for targeted treatment strategies for patients with GDM.

In conclusion, the present study assessed the function of miR-345-3p during GDM. The results revealed that miR-345-3p was abnormally expressed in patients with GDM, and high expression of miR-345-3p during GDM served a protective role by inhibiting placental trophoblast apoptosis, and promoting cell proliferation and migration via targeting BAK1. As the present study was only a preliminary study investigating the role of miR-345-3p during GDM, the possibility of using miR-345-3p to diagnose GDM requires further investigation. For example, the role of miR-345-3p/BAK1 during glucose metabolism and insulin signaling should be explored. In addition, the expression of miR-345-3p/BAK1 in patients with GDM prior and subsequent to insulin therapy requires further investigation. Furthermore, the effect of miR-345-3p/BAK1 during GDM should be investigated in vivo.

\section{Acknowledgements}

Not applicable.

\section{Funding}

No funding was received.

\section{Availability of data and materials}

The datasets used and/or analysed during the present study are available from the corresponding author on reasonable request.

\section{Authors' contributions}

YL contributed to study design, data collection, statistical analysis, data interpretation and manuscript preparation. JZ contributed to data collection, statistical analysis and manuscript preparation. All authors read and approved the final manuscript.

\section{Ethics approval and consent to participate}

The present study was approved by the Ethics Committee of Wuhan Children's Hospital (Wuhan Maternal and Child Healthcare Hospital). Written informed consent was obtained from all patients included in the present study.

\section{Patient consent for publication}

Not applicable.

\section{Competing interests}

The authors declare that they have no competing interests.

\section{References}

1. Metzger BE and Coustan DR: Summary and recommendations of the Fourth International Workshop-Conference on Gestational Diabetes Mellitus. The Organizing Committee. Diabetes Care 21 (Suppl 2): B161-B167, 1998.

2. Chiefari E, Arcidiacono B, Foti D and Brunetti A: Gestational diabetes mellitus: An updated overview. J Endocrinol Invest 40: 899-909, 2017.

3. Zhu Y and Zhang C: Prevalence of gestational diabetes and risk of progression to type 2 diabetes: A global perspective. Curr Diab Rep 16: 7, 2016.

4. Yuen L and Wong VW: Gestational diabetes mellitus: Challenges for different ethnic groups. World J Diabetes 6: 1024-1032, 2015.

5. Egan AM, Bogdanet D, Griffin TP, Kgosidialwa O, Cervar-Zivkovic M, Dempsey E, Allotey J, Alvarado F, Clarson C, Cooray SD, et al: A core outcome set for studies of gestational diabetes mellitus prevention and treatment. Diabetologia: Mar 20, 2020 (Epub ahead of print).

6. Tryggestad JB, Vishwanath A, Jiang S, Mallappa A, Teague AM Takahashi Y, Thompson DM and Chernausek SD: Influence of gestational diabetes mellitus on human umbilical vein endothelial cell miRNA. Clin Sci (Lond) 21: 1955-1967, 2016.

7. Gabbay-Benziv R and Baschat AA: Gestational diabetes as one of the 'great obstetrical syndromes'-The maternal, placental, and fetal dialog. Best Pract Res Clin Obstet Gynaecol 29: 150-155, 2015.

8. Winship A, Sorby K, Correia J, Rainczuk A, Yap J and Dimitriadis E: Interleukin-11 up-regulates endoplasmic reticulum stress induced target, PDIA4 in human first trimester placenta and in vivo in mice. Placenta 53: 92-100, 2017. 
9. Antoniotti GS, Coughlan M, Salamonsen LA and Evans J: Obesity associated advanced glycation end products within the human uterine cavity adversely impact endometrial function and embryo implantation competence. Hum Reprod. 33: 654-665, 2018.

10. Graham CH, Hawley TS, Hawley RG, MacDougall JR, Kerbel RS, Khoo N and Lala PK: Establishment and characterization of first trimester human trophoblast cells with extended lifespan. Exp Cell Res 206: 204-211, 1993.

11. Monfared YK, Ghadimi F, Foroughi F, Honardoost M, Hashemipour S, Sefidi F and Sarookhani MR: Determination and comparison miR135a in the serum between women with GDM, non-pregnant type 2 diabetes, healthy pregnant and control group. Middle East J Fam Med 2: 193-197, 2018

12. Eminaga O, Fries J, Woetzel F, Alakus H, Warnecke-Eberz U and Heidenreich A: MP66-12 the expression profiles of miR-210, miR-375, miR-378, miR-345, miR-143 miR-183 and miR-98 in the progression of prostate cancer from high-grade prostatic intraepithelial neoplasia to metastatic diseases. J Urology 4: e877, 2016.

13. Mou T, Xie F, Zhong P, Hua H, Lai L, Yang Q and Wang J: MiR-345-5p functions as a tumor suppressor in pancreatic cancer by directly targeting CCL8. Biomed Pharmacother 111: 891-900, 2019.

14. Danese E, Minicozzi AM, Benati M, Paviati E, Lima-Oliveira G Gusella M, Pasini F, Salvagno GL, Montagnana M and Lippi G: Reference miRNAs for colorectal cancer: Analysis and verification of current data. Sci Rep 7: 8413, 2017.

15. Ying X, Zhang W, Fang M, Zhang W, Wang C and Han L: miR-345-5p regulates proliferation, cell cycle, and apoptosis of acute myeloid leukemia cells by targeting AKT2. J Cell Biochem 2: 1620-1629, 2019

16. Wang SY, Shiboski S, Belair CD, Cooperberg MR, Simko JP, Stoppler H, Cowan J, Carroll PR and Blelloch R: MiR-19, miR-345, miR-519c-5p serum levels predict adverse pathology in prostate cancer patients eligible for active surveillance. PLoS One 6: e98597, 2014.

17. Tinay I, Tan M, Gui B, Werner L, Kibel AS and Jia L: Functional roles and potential clinical application of miRNA-345-5p in prostate cancer. Prostate 78: 927-937, 2018.

18. Srivastava SK, Bhardwaj A, Arora S, Tyagi N, Singh S, Andrews J, McClellan S, Wang B and Singh AP: MicroRNA-345 induces apoptosis in pancreatic cancer cells through potentiation of caspase-dependent and -independent pathways. Brit J Cancer 113: 660-668, 2015.

19. Tinay I, Gui B, Werner L, Rafiei S, Gelpi-Hammerschmidt F, Kibel A and Jia L: 24-Upregulation of microRNAs miR-9, miR-330-3p and miR-345 in prostate cancer. Eur Urol Suppl 15: e1286-e1287, 2016.

20. Siengdee P, Trakooljul N, Murani E, Schwerin M, Wimmers K and Ponsuksili S: MicroRNAs regulate cellular ATP levels by targeting mitochondrial energy metabolism genes during $\mathrm{C} 2 \mathrm{C} 12$ myoblast differentiation. PLoS One 10: e0127850, 2015

21. Livak KJ and Schmittgen TD: Analysis of relative gene expression data using realtime quantitative PCR and the 2(-Delta Delta C(T)) method. Methods 25: 402-408, 2001.

22. Uren RT, O'Hely M, Iyer S, Bartolo R, Shi MX, Brouwer JM, Alsop AE, Dewson G and Kluck RM: Disordered clusters of Bak dimers rupture mitochondria during apoptosis. Elife 6: pii: 19944, 2017.

23. Loegl J, Nussbaumer E, Cvitic S, Huppertz B, Desoye G and Hiden U: GDM alters paracrine regulation of feto-placental angiogenesis via the trophoblast. Lab Invest 97: 409-418, 2017.
24. Mohr AM and Mott JL: Overview of microRNA biology. Semin Liver Dis 35: 3-11, 2015.

25. Lu TX and Rothenberg ME: MicroRNA. J Allergy Clin Immunol 141: 1202-1207, 2018.

26. Li J, Song L, Zhou L, Wu J, Sheng C, Chen H, Liu Y, Gao S and Huang W: A MicroRNA signature in gestational diabetes mellitus associated with risk of macrosomia. Cell Physiol Bioche 1: 243-252, 2015.

27. Erener S, Marwaha A, Tan R, Panagiotopoulos C and Kieffer TJ: Profiling of circulating microRNAs in children with recent onset of type 1 diabetes. JCI Insight 4: e89656, 2017.

28. Silva VA, Polesskaya A, Sousa TA, Corrêa VM, André ND, Reis RI, Kettelhut IC, Harel-Bellan A and De Lucca FL: Expression and cellular localization of microRNA-29b and RAX, an activator of the RNA-dependent protein kinase (PKR), in the retina of streptozotocin-induced diabetic rats. Mol Vis 17: 2228-2240, 2011

29. Chen HY, Zhong X, Huang XR, Meng XM, You Y, Chung AC and Lan HY: MicroRNA-29b inhibits diabetic nephropathy in $\mathrm{db} / \mathrm{db}$ mice. Mol Ther 22: 842-853, 2014.

30. Knöfler M, Haider S, Saleh L, Pollheimer J, Gamage TKJB and James J: Human placenta and trophoblast development: Key molecular mechanisms and model systems. Cell Mol Life Sci 76: 3479-3496, 2019

31. Pollheimer J, Vondra S, Baltayeva J, Beristain AG and Knöfler M: Regulation of placental extravillous trophoblasts by the maternal uterine environment. Front Immunol 9: 2597, 2018.

32. Haider S, Meinhardt G, Saleh L, Kunihs V, Gamperl M, Kaindl U, Ellinger A, Burkard TR, Fiala C, Pollheimer J, et al: Self-renewing trophoblast organoids recapitulate the developmental program of the early human placenta. Stem Cell Reports 11: 537-551, 2018.

33. Wang X, Peng S, Cui K, Hou F, Ding J, Li A, Wang M and Geng L: MicroRNA-576-5p enhances the invasion ability of trophoblast cells in preeclampsia by targeting TFAP2A. Mol Genet Genomic Med 8: e1025, 2020

34. Baines KJ and Renaud SJ: Transcription factors that regulate trophoblast development and function. Prog Mol Biol Transl Sci 145: 39-88, 2017.

35. Aplin JD: Developmental cell biology of human villous trophoblast: Current research problems. Int J Dev Biol 54: 323-329, 2010.

36. Meekins JW, Pijnenborg R, Hanssens M, McFadyen IR and van Asshe A: A study of placental bed spiral arteries and trophoblast invasion in normal and severe pre-eclamptic pregnancies. Brit J Obstet Gynaec 8: 669-674, 1994.

37. Mayama R, Izawa T, Sakai K, Suciu N and Iwashita M: Improvement of insulin sensitivity promotes extravillous trophoblast cell migration stimulated by insulin-like growth factor-I. Endocr J 60: 359-368, 2013.

38. Aires MB and Anne Carolline Veríssimo dos Santos: Effects of maternal diabetes on trophoblast cells. World J Diabetes 6: $338-344,2015$.

This work is licensed under a Creative Commons Attribution-NonCommercial-NoDerivatives 4.0 International (CC BY-NC-ND 4.0) License. 